\title{
Metal Halide Scaffolded Assemblies of Organic Molecules with Enhanced Emission and Room Temperature Phosphorescence.
}

Maya Chaaban ${ }^{\dagger}$, Azza Ben-Akacha ${ }^{\dagger}$, Michael Workul, Sujin Lee ${ }^{\dagger}$, Jennifer Neu ${ }^{\S}$ Xinsong Lin†, J.S. Raaj Vellore Winfred ${ }^{\dagger}$, Cordell J. Delzer ${ }^{\ddagger}$, Jason P. Hayward ${ }^{\ddagger}$, Mao-Hua Du ${ }^{o}$, Theo Siegrist ${ }^{\sharp \#}$, and Biwu Ma ${ }^{\dagger / / *}$

${ }^{\dagger}$ Department of Chemistry and Biochemistry, Florida State University, Tallahassee, FL 32306, USA.

"Materials Science and Engineering Program, Florida State University, Tallahassee, FL 32306, USA.

$\S$ National High Magnetic Field Laboratory, Florida State University, Tallahassee, Florida 32310, USA.

${ }^{\ddagger}$ Department of Nuclear Engineering, University of Tennessee, Knoxville, TN 37996, USA. ${ }^{\circ}$ Materials Science \& Technology Division, Oak Ridge National Laboratory, Oak Ridge, Tennessee 37831, USA.

${ }^{\#}$ Department of Chemical and Biomedical Engineering, FAMU-FSU College of Engineering, Tallahassee, FL 32310, USA

\section{Materials and Synthesis}

Materials: Lead chloride ( $\mathrm{PbCl}_{2}$, 99.99\%), lead(II) bromide ( $\left.\mathrm{PbBr} 2,99.99 \%\right)$, Benzo[h]quinoline (97\%), Hydrochloric acid (37\%), Hydrobromic acid (48\%) were purchased from Sigma Aldrich and were used without further purification.

Synthesis of (BZQ)Pb2 $\mathbf{C l}_{5}$ : Lead chloride $(0.35 \mathrm{mmol}, 9 \mathrm{mg})$ and benzo[h]quinoline (0.17, 31 $\mathrm{mg}$ ) were added to a round bottom flask with $10 \mathrm{~mL}$ of water and $1 \mathrm{~mL}$ of $\mathrm{HCl}$. The mixture was stirred and heated at $100^{\circ} \mathrm{C}$ until all the reactants dissolved. Upon cooling the mixture slowly, small plate-like crystals precipitate. The crystals were washed with water and acetone and dried under reduced pressure. The yield was calculated at $64 \%$.

Synthesis of (BZQ)Pb2Br5: Lead bromide $(0.35 \mathrm{mmol}, 127 \mathrm{mg})$ and benzo[h]quinoline $(0.17,31$ $\mathrm{mg}$ ) were added to a round bottom flask with $10 \mathrm{~mL}$ of water and $1 \mathrm{~mL}$ of $\mathrm{HBr}$. The mixture was stirred and heated at $100^{\circ} \mathrm{C}$. Upon cooling the mixture, small plate-like crystals precipitate. The crystals were washed with water and acetone and dried under reduced pressure. The yield was calculated at $\sim 67 \%$.

Synthesis of (BZQ)Cl: Benzo[h]quinoline (1 eq.) were dissolved in ethanol in an ice bath, 1.1 equivalent of $\mathrm{HCl}$ was added. The mixture was stirred, and grayish-white powder precipitated after $10 \mathrm{~min}$. The reaction mixture was filtered, and the product was washed with ethanol and 
diethyl ether and dried under reduced pressure. Single crystals of (BZQ)Cl were grown by solvent diffusion of diethyl ether into a concentrated solution of (BZQ)Cl in dimethylformamide (DMF). The crystals were transparent colorless plates.

Synthesis of (BZQ)Br: Benzo[h]quinoline (1 eq.) were dissolved in ethanol in an ice bath, 1.1 equivalent of $\mathrm{HBr}$ was added. The mixture was stirred for 2 hours until the formation of a white precipitate. The reaction mixture was filtered, and the product was washed with ethanol and diethyl ether and dried under reduced pressure. Single crystals of (BZQ)Br were grown by solvent diffusion of diethyl ether into a concentrated solution of (BZQ)Br in dimethylformamide (DMF). The crystals were transparent pale yellowish plates.

\section{Characterizations of structural and photophysical properties}

Single crystal X-ray crystallography: The single crystal structures were determined using Rigaku XtaLAB Synergy-S diffractometer equipped with a HyPix-6000HE Hybrid Photon Counting (HPC) detector and dual Mo and Cu microfocus sealed X-ray sources. The crystals were mounted in cryoloop with Paratone-N oil. The data were collected at 100.00(10) K. The structures were solved using Olex2 software where $\mathrm{XT}^{1}$ structure solution program using Intrinsic Phasing and the $\mathrm{XL}^{2}$ refinement package using Least Squares minimization were employed in solving and refining the structures, respectively. Table S1 summarizes the refinement details and the resulting factors.

Powder X-ray crystallography: The PXRD analysis was performed on Rigaku SmartLab powder X-Ray diffractometer with the PhotonMax high-flux $9 \mathrm{~kW}$ rotating anode X-ray source coupled with a 1D measurement modes. The diffraction pattern was scanned over the angular range of 5-60 degrees (2 $\theta$ ) with a step size of 0.05 at room temperature. Simulated powder patterns were calculated in the Mercury software from the corresponding CIF files.

Thermogravimetric analysis (TGA): TGA was conducted on the Q50 TGA system. The samples were heated from room temperature to $800{ }^{\circ} \mathrm{C}$ at a rate of $5{ }^{\circ} \mathrm{C} / \mathrm{min}$, under an argon flux of $30 \mathrm{ml} / \mathrm{min}$.

Photoluminescence studies: The emission and excitation spectra were measured at room temperature and at $77 \mathrm{~K}$ (using liquid $\mathrm{N}_{2}$ ) on a FS5 spectrofluorometer (Edinburgh Instruments).

Time-resolved photoluminescence studies: The lifetimes in the range of nanosecond were evaluated at room temperature and at $77 \mathrm{~K}$ (using liquid $\mathrm{N}_{2}$ ) using time-correlated single photon counting on a FS5 spectrofluorometer (Edinburgh Instruments) with data collection for 10000 counts. The samples were excited by an Edinburgh EPL-365 picosecond pulsed diode laser and the emission counts was monitored at corresponding emission maximums. The Long lifetime in the range of millisecond were measured using the Edinburgh Instruments LP980-KS laser flash photolysis spectrometer using an Nd:YAG pump laser as the excitation source and Hamamatsu R928 photomultiplier detector operating in multichannel scaling MCS mode as a detector. T25he emission counts were monitored at the corresponding emission maximums, and the data was collected to 10000 counts. The long-lived lifetime was simulated by bi-exponential and average decay lifetimes were obtained through the following equation.

$\tau=(a 1 * \tau 1 * \tau 1+a 2 * \tau 2 * \tau 2) /(a 1 * \tau 1+a 2 * \tau 2)$ 
Photoluminescence quantum efficiency (PLQE): The PLQEs were measured using a Hamamatsu Quantaurus-QY Spectrometer (Model C11347-11) equipped with a xenon lamp, integrated sphere sample chamber, and CCD detector. The PLQEs were calculated by the equation: $\eta Q E=I S /(E R-E S)$, in which IS represents the luminescence emission spectrum of the sample, ER is the spectrum of the excitation light from the empty integrated sphere (without the sample), and ES is the excitation spectrum for exciting the sample.

Computational Methods: The calculations are based on density functional theory (DFT) as implemented in the VASP code. ${ }^{3}$ The kinetic energy cutoff of the plane-wave basis is $400 \mathrm{eV}$. The projector augmented wave method was used to describe the interaction between ions and electrons. ${ }^{4}$ The lattice parameters were fixed at the experimentally measured values while the atomic positions were optimized until the force on each ion is less than $0.02 \mathrm{eV} / \AA$. The electronic band structure and density of states (DOS) of (BZQ) $\mathrm{Pb}_{2} \mathrm{X}_{5}(\mathrm{X}=\mathrm{Cl}, \mathrm{Br}$ ) were calculated using Perdew-Burke-Ernzerhof (PBE) exchange-correlation functional. ${ }^{5}$ The band gaps were further corrected using the hybrid PBE0 functional, which has a $25 \%$ non-local Fock exchange. The inclusion of a fraction of Fock exchange significantly improves the calculation of the bandgap energy. ${ }^{6-8}$

Hirshfeld Surfaces and 2D Fingerprint Plots: The Hirshfeld surfaces and 2D fingerprint plots for the different materials were calculated by using Crystal Explorer 17.5. ${ }^{9}$ First, the CIF files were imported into the software the $\mathrm{BZQ}^{+}$cations were selected. In the window of surface generation, the option "Hirshfeld" was selected for the surface, and its property was set as "None". The option "High (Standard)" was chosen for the resolution. After generating a Hirshfeld surface, "Surface property" on the bottom right of the window was set as "d norm" by default. Additionally, in the window of $2 \mathrm{D}$ fingerprint plots, the isovalue was 0.5 by default. The type was defaulted to "di vs de". The option "Expanded" was selected for the range, and "By Element" was selected for the filter. 
Table S1. Crystallographic data for (BZQ)Cl, (BZQ)Pb2 $\mathrm{Cl}_{5}$, (BZQ)Br, and (BZQ)Pb2 $\mathrm{Br}_{5}$.

\begin{tabular}{|c|c|c|c|c|}
\hline Compound & (BZQ)Cl & $\left(\mathrm{BZQ} \mathrm{Pb}_{2} \mathrm{Cl}_{5}\right.$ & (BZQ)Br & $\left(\mathrm{BZQ} \mathrm{Pb}_{2} \mathrm{Br}_{5}\right.$ \\
\hline CCDC & 2092022 & 2092024 & 2092025 & 2092023 \\
\hline Chemical formula & $\mathrm{C}_{13} \mathrm{H}_{10} \mathrm{NCl}$ & $\mathrm{C}_{13} \mathrm{H}_{10} \mathrm{NPb}_{2} \mathrm{Cl}_{5}$ & $\mathrm{C}_{13} \mathrm{H}_{10} \mathrm{BrN}$ & $\mathrm{C}_{13} \mathrm{H}_{10} \mathrm{NPb}_{2} \mathrm{Br}_{5}$ \\
\hline Formula weight & 215.67 & 771.85 & 260.13 & 994.15 \\
\hline Temperature (K) & $99.9(3)$ & $100(1)$ & $100.6(10)$ & $100.0(10)$ \\
\hline Crystal system & Monoclinic & Monoclinic & Monoclinic & Monoclinic \\
\hline Space group & $\mathrm{P} 21 / \mathrm{c}$ & $\mathrm{P} 21 / \mathrm{c}$ & $\mathrm{P} 2{ }_{1} / \mathrm{C}$ & $\mathrm{P} 21 / \mathrm{c}$ \\
\hline$a(\AA)$ & $17.5101(2)$ & $13.0000(2)$ & $17.51100(10)$ & $13.1305(2)$ \\
\hline$b(\AA)$ & $11.09440(10)$ & $11.5679(2)$ & $11.36730(10)$ & $11.9184(2)$ \\
\hline$c(\AA)$ & $16.4094(2)$ & 11.6620(2) & $16.56155(10)$ & 12.0846(3) \\
\hline$\alpha\left({ }^{0}\right)$ & 90 & 90 & 90 & 90 \\
\hline$\beta\left({ }^{0}\right)$ & $106.9460(10)$ & $107.253(2)$ & 105.0998(7) & $105.876(2)$ \\
\hline$\gamma\left({ }^{0}\right)$ & 90 & 90 & 90 & 90 \\
\hline Volume $\left(\AA^{3}\right)$ & $3049.34(6)$ & 1674.85(5) & 3182.81(4) & 1819.04(6) \\
\hline$Z$ & 12 & 4 & 12 & 4 \\
\hline$\rho_{\text {cal }}\left(\mathrm{g} / \mathrm{cm}^{3}\right)$ & 1.409 & 3.061 & 1.629 & 3.630 \\
\hline Crystal size $\left(\mathrm{mm}^{3}\right)$ & $\begin{array}{c}0.195 \times 0.206 \\
\times 0.213\end{array}$ & $\begin{array}{c}0.084 \times 0.296 \times \\
0.428\end{array}$ & $\begin{array}{c}0.089 \times 0.114 \times \\
0.46\end{array}$ & $\begin{array}{c}0.037 \times 0.073 \times \\
0.075\end{array}$ \\
\hline$\mu\left(\mathrm{mm}^{-1}\right)$ & 2.986 & 20.865 & 4.957 & 48.366 \\
\hline $\mathrm{F}(000)$ & 1344.0 & 1376.0 & 1560.0 & 1736.0 \\
\hline $2 \theta$ range $\left(^{\circ}\right)$ & $\begin{array}{c}5.276 \text { to } \\
152.79\end{array}$ & 3.28 to 52.74 & 5.228 to 155.45 & 7 to 155.602 \\
\hline Reflection collected & 30025 & 50949 & 40813 & 40330 \\
\hline Independent reflections & 6247 & 3436 & 6721 & 3716 \\
\hline $\begin{array}{c}\text { Data/restraints/ } \\
\text { parameters }\end{array}$ & $6247 / 0 / 406$ & $3436 / 0 / 190$ & $6721 / 0 / 406$ & $3716 / 0 / 190$ \\
\hline$R_{\text {int }}$ & 0.0362 & 0.1095 & 0.0382 & 0.1038 \\
\hline GOF & 1.058 & 1.052 & 1.092 & 1.135 \\
\hline $\begin{array}{l}\text { Final } R \text { indices } \\
{[\mathrm{I}>2 \sigma(\mathrm{I})]}\end{array}$ & $\begin{array}{c}R_{1}=0.0328 \\
w R_{2}=0.0871\end{array}$ & $\begin{array}{c}R_{1}=0.0267 \\
w R_{2}=0.0674\end{array}$ & $\begin{array}{l}R_{1}=0.0359 \\
w R_{2}=0.0915\end{array}$ & $\begin{array}{l}R_{1}=0.0403 \\
w R_{2}=0.1113\end{array}$ \\
\hline $\begin{array}{l}\text { Final } R \text { indices [all } \\
\text { data] }\end{array}$ & $\begin{array}{c}R_{1}=0.0360 \\
w R_{2}=0.0893\end{array}$ & $\begin{array}{c}R_{1}=0.0300 \\
w R_{2}=0.0692\end{array}$ & $\begin{array}{c}R_{1}=0.0363 \\
w R_{2}=0.0918\end{array}$ & $\begin{array}{c}R_{1}=0.0431 \\
w R_{2}=0.1139\end{array}$ \\
\hline
\end{tabular}




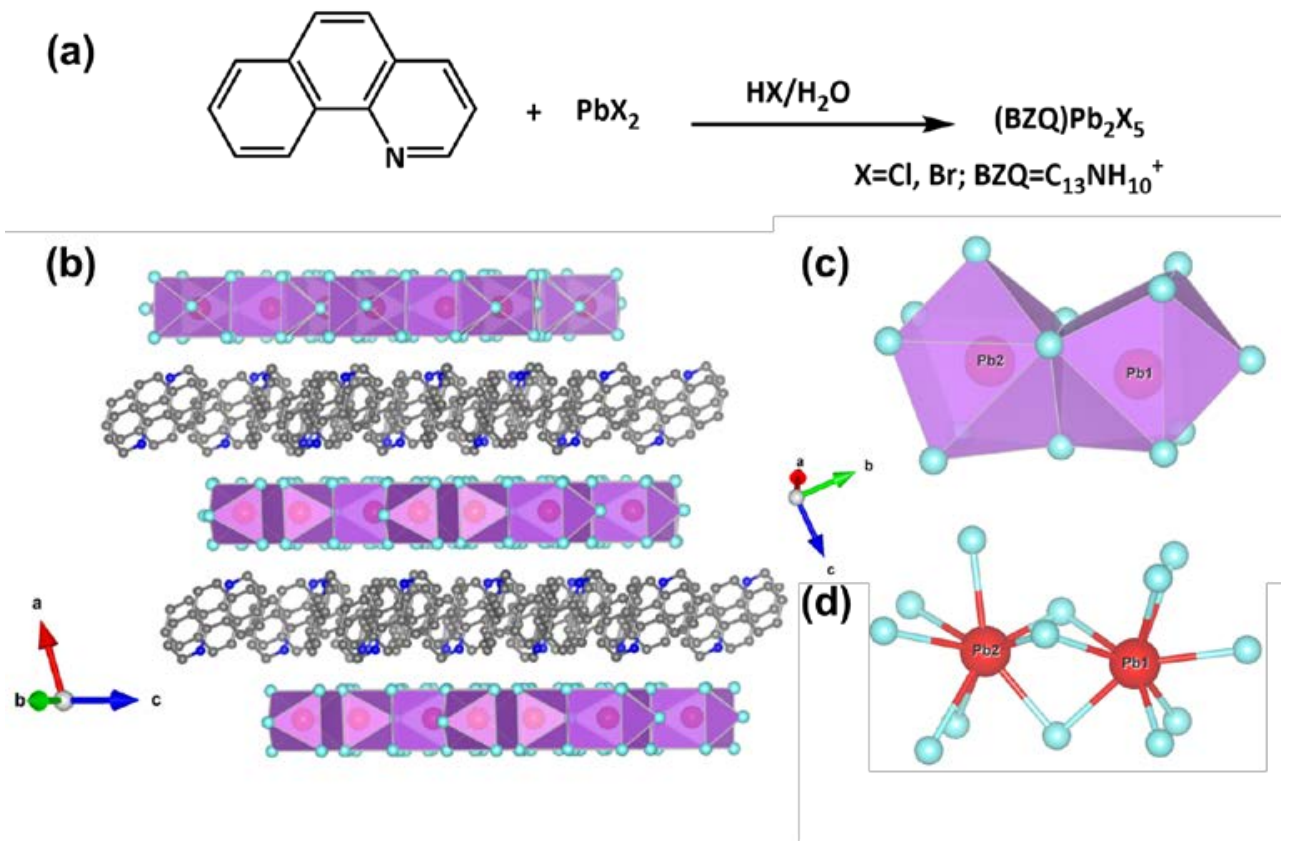

Figure $\mathrm{S1}$ a) Synthetic scheme of (BZQ) $\mathrm{Pb}_{2} \mathrm{X}_{5}$. b) View of the single crystal structure of $(\mathrm{BZQ}) \mathrm{Pb}_{2} \mathrm{Cl}_{5}$. c) View of face-sharing decahedra in (BZQ) $\mathrm{Pb}_{2} \mathrm{Cl}_{5}$. d) Ball-and-stick model of an individual the face-sharing decahedra. ( $\mathrm{Pb}$ : red, $\mathrm{Cl}$ : light blue, $\mathrm{N}$ : blue, $\mathrm{C}$ : gray, hydrogen atoms are omitted for clarity).

(a)

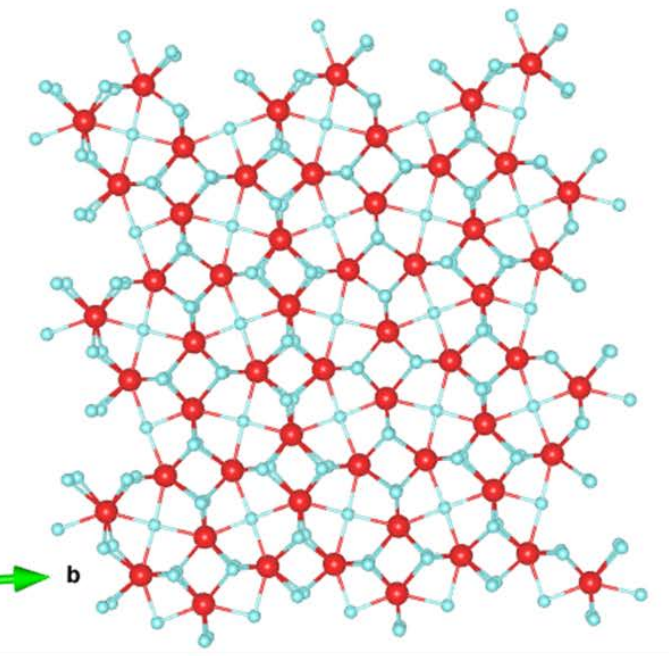

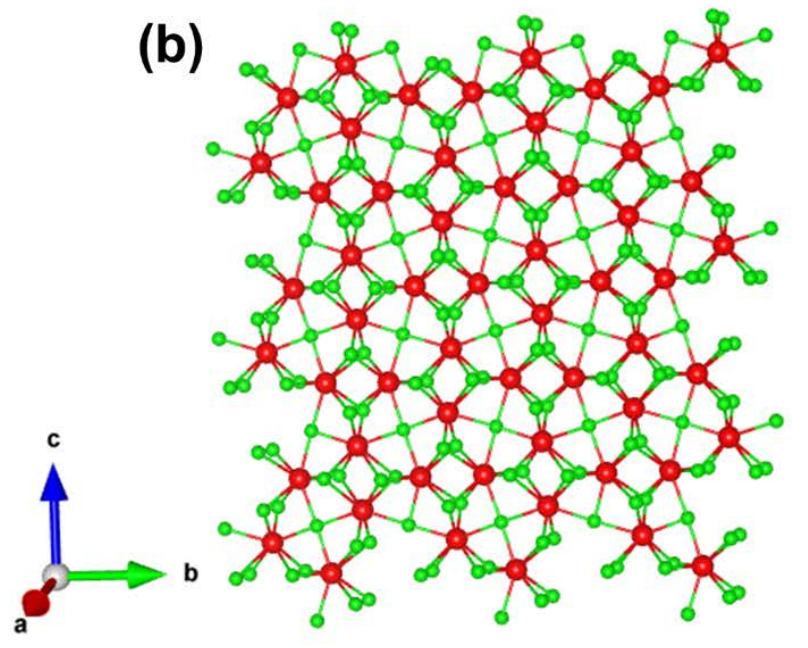

Figure S2. Views of $\mathrm{Pb}_{2} \mathrm{X}_{5}-$ layers in the single crystal structures of a) (BZQ) $\mathrm{Pb}_{2} \mathrm{Cl}_{5}$ and b) (BZQ)Pb2Brs. (Pb: red, Cl: light blue, Br: green) 

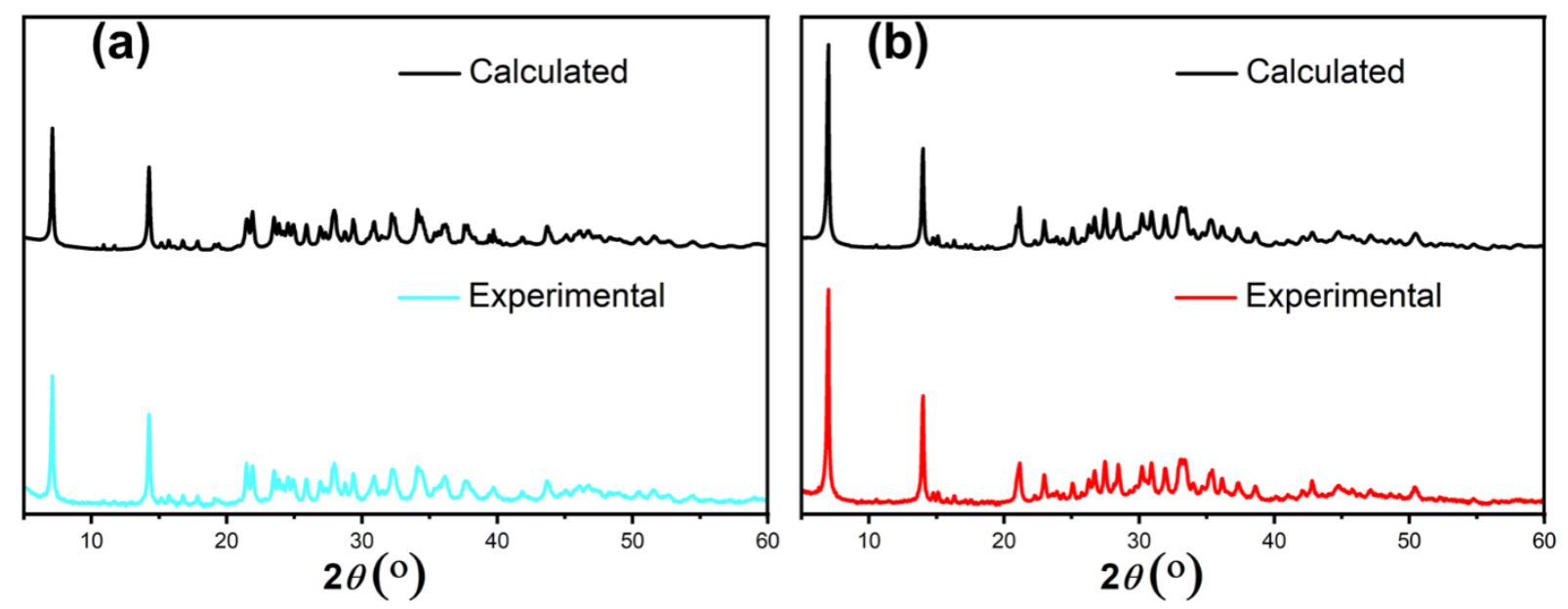

Figure S3. Comparison between the experimental and simulated PXRD patterns of a) (BZQ)Pb2 $\mathrm{Cl}_{5}$ and b) (BZQ)Pb2Br5.

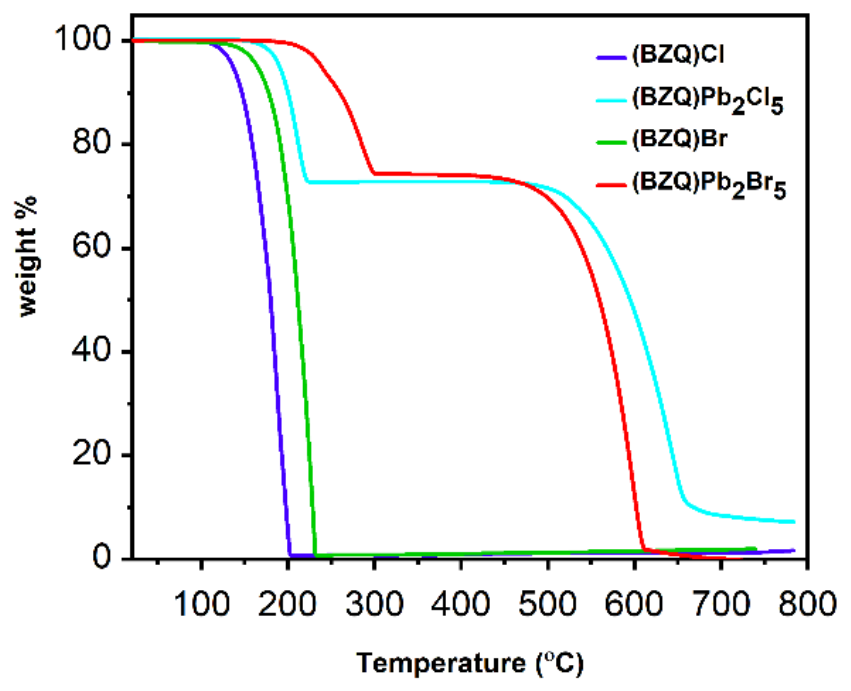

Figure S4. TGA analysis of the different compounds. 

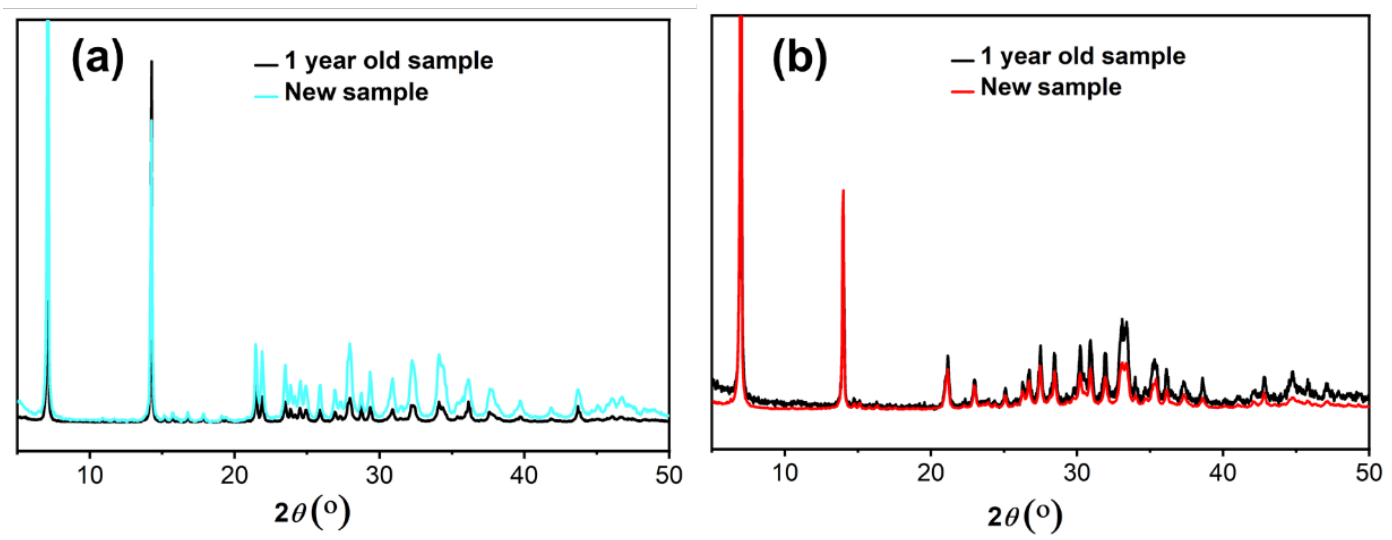

Figure S5. Comparison between the PXRD patterns of new and 1 year old samples of a) (BZQ) $\mathrm{Pb}_{2} \mathrm{Cl}_{5}$ b) (BZQ)Pb ${ }_{2} \mathrm{Br}_{5}$.
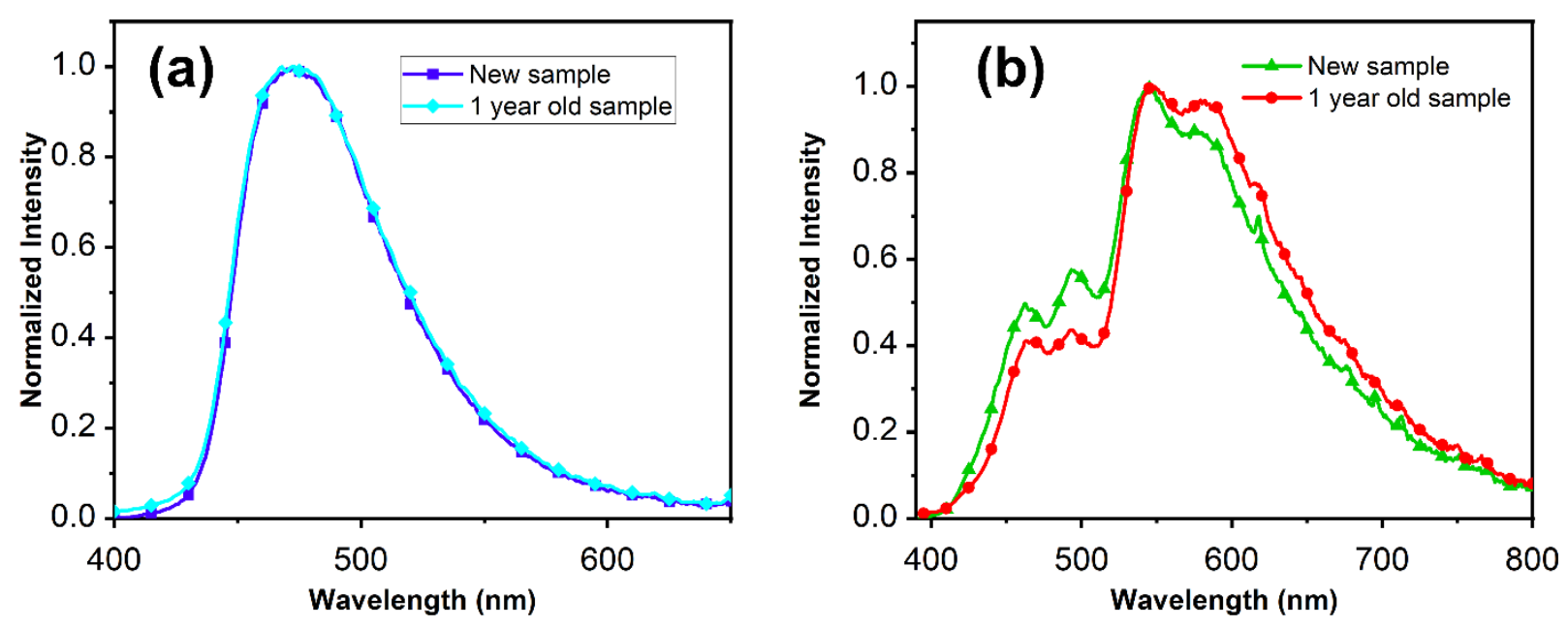

Figure S6. Comparison between the PL spectra of new and 1 year old samples of a) (BZQ) $\mathrm{Pb}_{2} \mathrm{Cl}_{5}$ and b) (BZQ) $\mathrm{Pb}_{2} \mathrm{Br}_{5}$. 

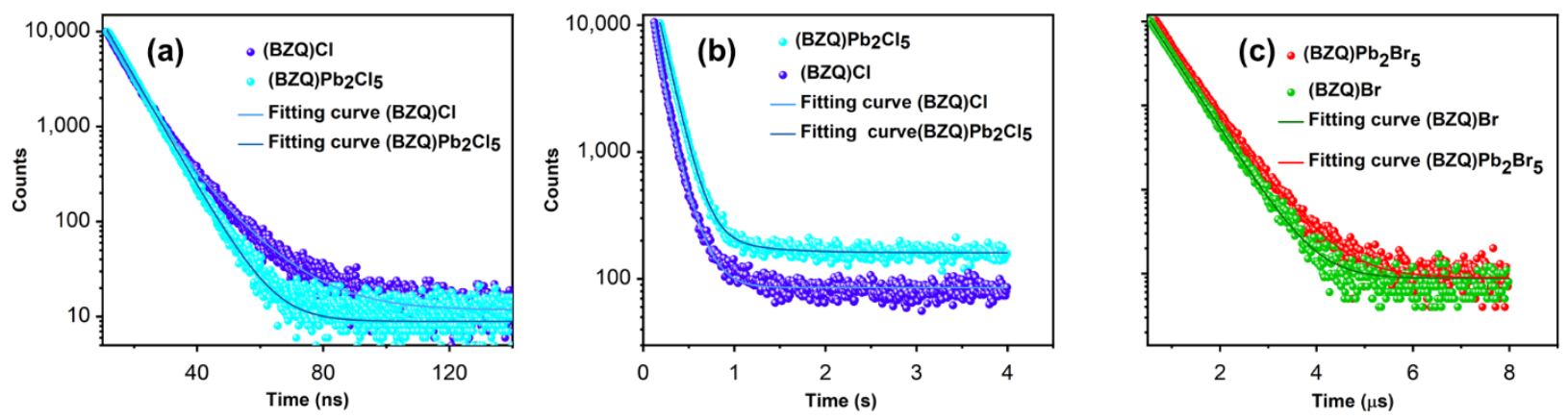

Figure S5. Emission decay curves at $77 \mathrm{~K}$ of a) (BZQ)Cl and (BZQ) $\mathrm{Pb}_{2} \mathrm{Cl}_{5}$ monitored at $450 \mathrm{~nm}$ b) (BZQ)Cl and (BZQ)Pb2 $\mathrm{Cl}_{5}$ monitored at $\left.530 \mathrm{~nm} \mathrm{c}\right)(\mathrm{BZQ}) \mathrm{Pb}_{2} \mathrm{Br}_{5}$ monitored at $560 \mathrm{~nm}$.

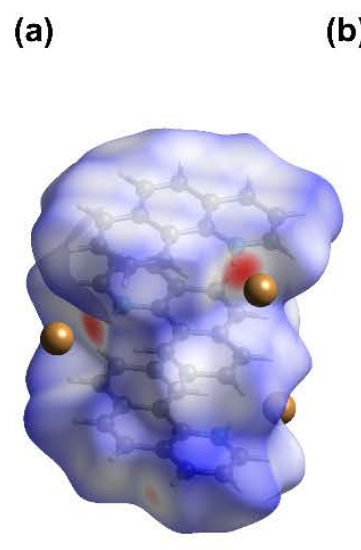

(b)
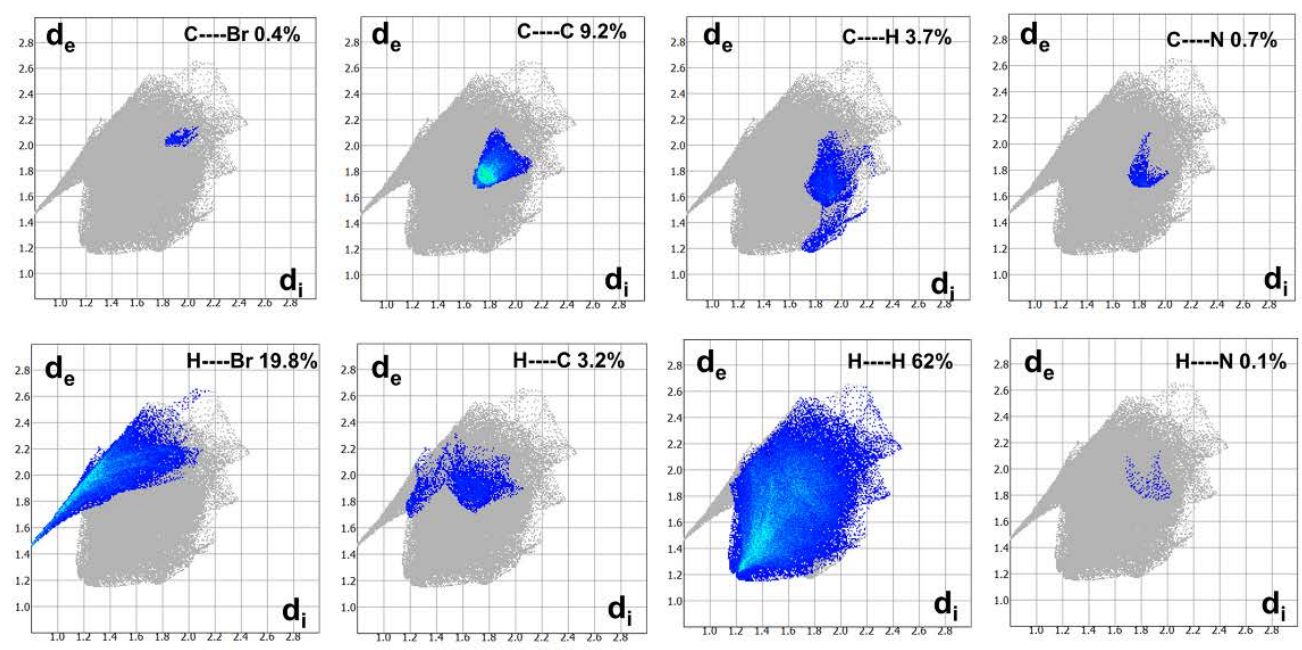

Figure S6. a) Image showing Hirshfeld surfaces for the $\mathrm{BZQ}^{+}$cation in (BZQ)Br. The Hirshfeld surface is a series of isosurfaces with three colors: red, white, and blue regions. These regions denote intermolecular contact distances that are shorter (red), equal (white), and longer (blue) than the van der Waals distances. b) 2D fingerprint plots and relative contributions (in \%) to the Hirshfeld surface areas for different intermolecular contacts in (BZQ)Br. 
(a)

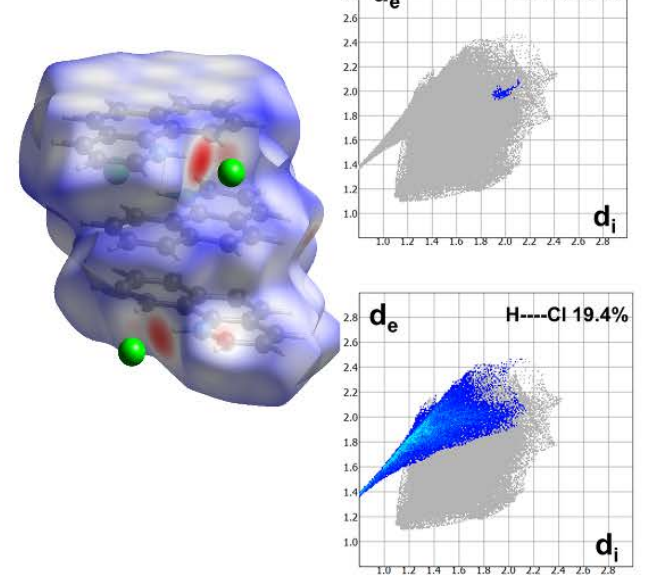

(b)

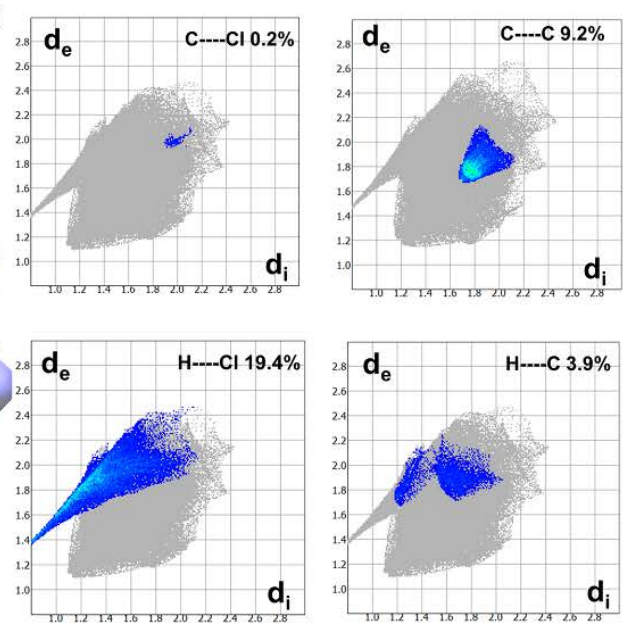

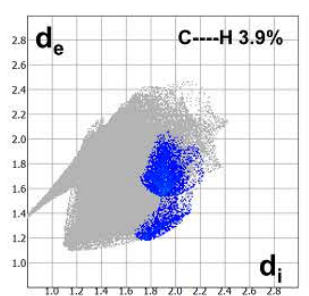
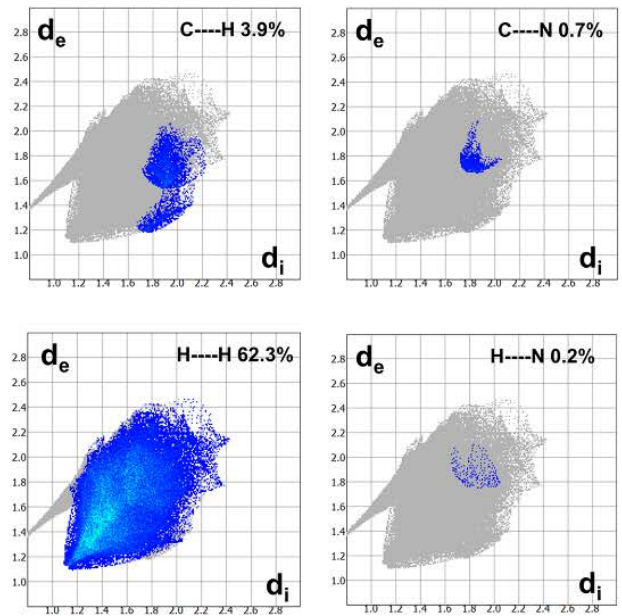

Figure S7. a) Image showing Hirshfeld surfaces for the $\mathrm{BZQ}^{+}$cation in (BZQ)Cl. The Hirshfeld surface is a series of isosurfaces with three colors: red, white, and blue regions. These regions denote intermolecular contact distances that are shorter (red), equal (white), and longer (blue) than the van der Waals distances. b) 2D fingerprint plots and relative contributions (in \%) to the Hirshfeld surface areas for different intermolecular contacts in (BZQ)Cl.

(a)

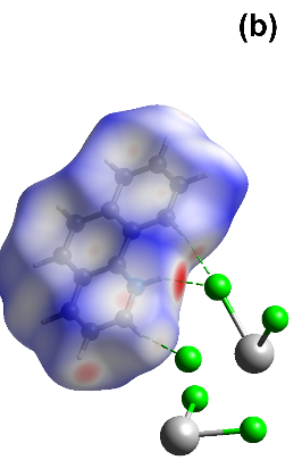

(b)
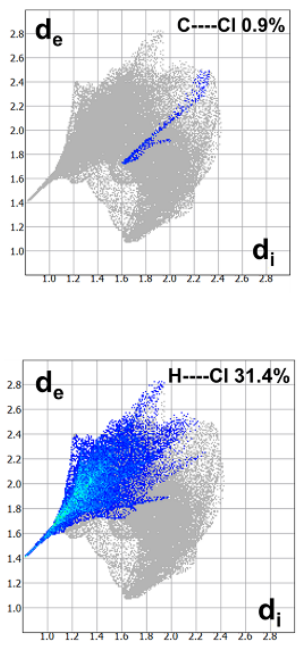
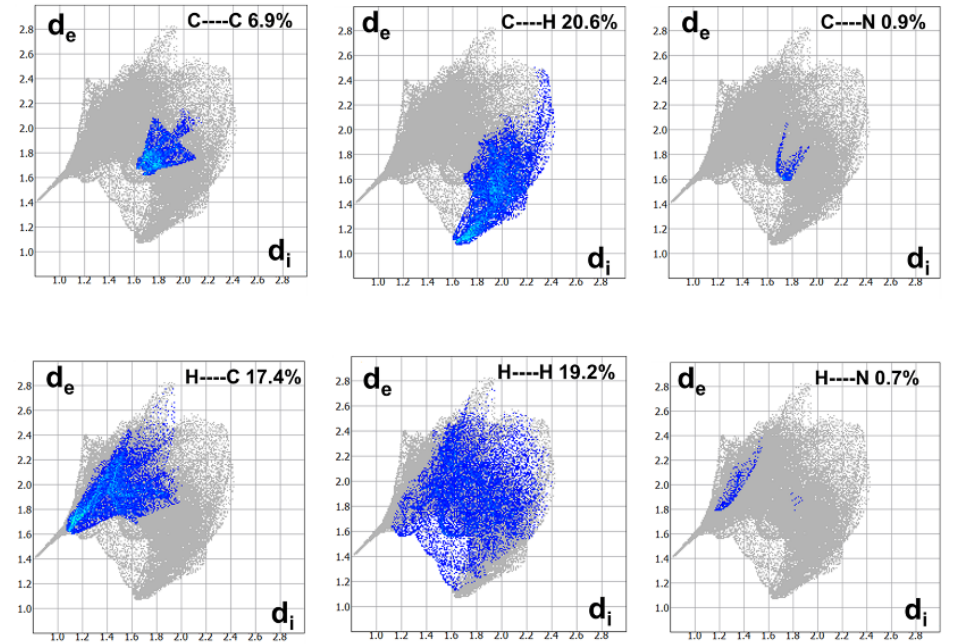

Figure S8. a) Image showing Hirshfeld surfaces for the $\mathrm{BZQ}^{+}$cation in (BZQ) $\mathrm{Pb}_{2} \mathrm{Cl}_{5}$. The Hirshfeld surface is a series of isosurfaces with three colors: red, white, and blue regions. These regions denote intermolecular contact distances that are shorter (red), equal (white), and longer (blue) than the van der Waals distances. b) 2D fingerprint plots and relative contributions (in \%) to the Hirshfeld surface areas for different intermolecular contacts in (BZQ) $\mathrm{Pb}_{2} \mathrm{Cl}_{5}$. 


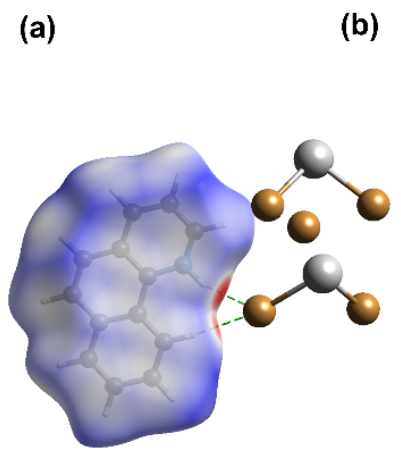

(b)
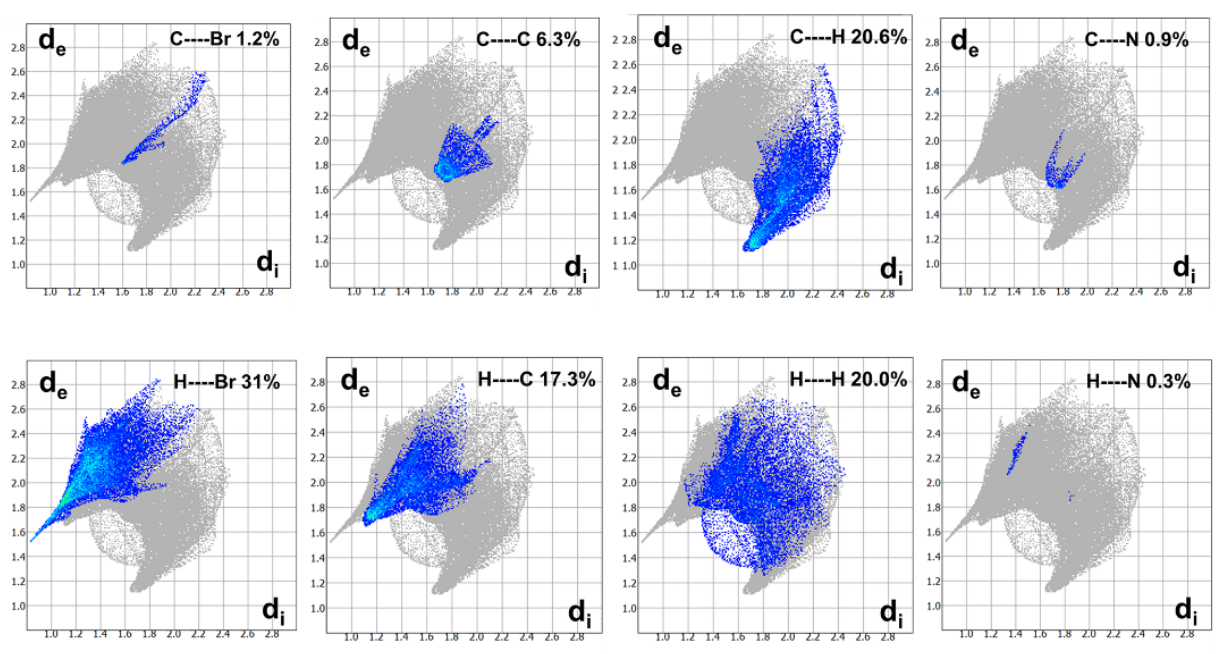

Figure S9. a) Image showing Hirshfeld surfaces for the $\mathrm{BZQ}^{+}$cation in (BZQ) $\mathrm{Pb}_{2} \mathrm{Br}_{5}$. The Hirshfeld surface is a series of isosurfaces with three colors: red, white, and blue regions. These regions denote intermolecular contact distances that are shorter (red), equal (white), and longer (blue) than the van der Waals distances. b) 2D fingerprint plots and relative contributions (in \%) to the Hirshfeld surface areas for different intermolecular contacts in (BZQ) $\mathrm{Pb}_{2} \mathrm{Br} 5$.

1. Sheldrick, G. SHELXT - Integrated space-group and crystal-structure determination. Acta Crystallogr. A 2015, 71 (1), 3-8.

2. $\quad$ Sheldrick, G. A short history of SHELX. Acta Crystallogr. A 2008, 64 (1), 112-122.

3. Kresse, G.; Furthmüller, J. Efficiency of ab-initio total energy calculations for metals and semiconductors using a plane-wave basis set. Comput. Mater. Sci. 1996, 6 (1), 15-50.

4. $\quad$ Kresse, G.; Joubert, D. From ultrasoft pseudopotentials to the projector augmented-wave method. Phys. Rev. B 1999, 59 (3), 1758-1775.

5. $\quad$ Perdew, J. P.; Burke, K.; Ernzerhof, M. Generalized gradient approximation made simple. Phys. Rev. Lett. 1996, 77 (18), 3865.

6. $\quad$ Perdew, J. P.; Ernzerhof, M.; Burke, K. Rationale for mixing exact exchange with density functional approximations. J. Chem. Phys. 1996, 105 (22), 9982-9985.

7. $\quad$ Paier, J.; Marsman, M.; Hummer, K.; Kresse, G.; Gerber, I. C.; Ángyán, J. G. Screened hybrid density functionals applied to solids. J. Chem. Phys. 2006, 124 (15), 154709.

8. Heyd, J.; Scuseria, G. E.; Ernzerhof, M. Hybrid functionals based on a screened Coulomb potential. J. Chem. Phys. 2003, 118 (18), 8207-8215.

9. $\quad$ Spackman, P. R.; Turner, M. J.; McKinnon, J. J.; Wolff, S. K.; Grimwood, D. J.; Jayatilaka, D.; Spackman, M. A. CrystalExplorer: a program for Hirshfeld surface analysis, visualization and quantitative analysis of molecular crystals. J. Appl. Crystallogr. 2021, 54 (3), 1006-1011. 